\title{
Radiation therapy in chronic hidradenitis suppurativa: case report
}

\author{
Pitchaya Sakyanun ${ }^{1}$, Thirada Vongvanichvathana ${ }^{2}$, Prasert Lertsanguansinchai ${ }^{2}$ \\ 'Department of Radiation Oncology, Phramongkutklao Hospital, Bangkok, Thailand \\ ${ }^{2}$ Department of Radiation Oncology, Wattanosoth Cancer Hospital, Bankok, Thailand
}

Received: June 2, 2021

Revised: October 13, 2021

Accepted: November 1, 2021

Correspondence:

Pitchaya Sakyanun

Department of Radiation Oncology,

Phramongkutklao Hospital, 315

Ratchawithi Rd, Thung Phaya Thai,

Ratchathewi, Bangkok 10400,

Thailand.

Tel: +6623103000

E-mail:Sakyanun.pitchaya@gmail.

com

ORCID:

https://orcid.org/0000-0001-9687-4623
A 26-year-old female presented recurrent painful, carbuncles at both axillae for 10 years. It caused offensive odor and scar. Tissue diagnosis was chronic hidradenitis suppurativa. She was treated using antibiotics followed by multiple excisions with drainage but showed no improvement. Isotretinoin provided no benefit. She was socially isolated and experienced lower quality of life. Consequently, she was sent for radiation therapy. Computed tomography simulation was performed revealing an ulcer with deep chronic pus tracts at her axilla. Three-dimensional conformal radiation therapy was provided with $6 \mathrm{MV}$ photon (7.5 Gy in 3 fractions) covering all ulcers and pus tracts. On the last day of radiation therapy, carbuncles and wounds at the left axilla exhibited much improvement without pus. Three months follow-up showed much improvement of the lesions. The skin was smoother without pus or odor. Radiation therapy was confirmed one treatment option for chronic hidradenitis suppurativa.

Keywords: Radiotherapy, Hidradenitis suppurativa, Treatment outcome, Axillary disease, Skin diseases

\section{Introduction}

Hidradenitis suppurativa, also known as acne inversa or Verneuil's disease, is a chronic inflammatory skin disease caused by recurring chronic, painful and suppurative lesions. Symptoms of chronic hidradenitis suppurativa manifest as painful, deep-seated, inflamed lesions, represented in nodules, sinus tracts, and no comedone abscesses in the flexural regions, i.e., the axillae, inframammary folds, and inguinal, perineal and intergluteal areas, etc. Some symptoms like stinging, burning, pain, pruritus, hyperhidrosis and heat may appear before developing nodule lesions. The diagnosis of hidradenitis suppurativa is generally made clinically. A delayed diagnosis is common, because the disease is often mistaken for other infections. One study showed a median delay of about 12 years [1].

On physical examination, inflamed and noninflamed nodules, sinus tracts and abscesses in the axillary, inguinal, and anogenital regions may be found. The lesions may extend beyond these areas and appear around the anus, on the buttocks, or on the breasts among females. In atypical or refractory cases, additional investigations may be helpful. Biopsies and bacterial cultures are performed. Routine bacterial cultures from the lesions in hidradenitis suppurativa are frequently negative; nevertheless, the flares are occasionally associated with superinfection involving a range of bacteria, including Staphylococcus aureus [2]. Ultrasonography may help in assessing whether the extensive surgery is planned, by identifying subclinical extension of the lesions [3].

Hidradenitis suppurativa is divided in three categories using the Hurley staging system [4]. Stage I consists of solitary or multiple isolated abscesses without scarring or sinus tract formation. Stage II is characterized by single or multiple recurrent abscesses with widely separated lesions and sinus tract formation. Stage III is characterized by diffuse involvement across a region with multiple 
interconnected sinus tracts and abscesses [5] (Fig. 1). Treatment in hidradenitis suppurativa is categorized as preventive, pharmacologic, surgical, local therapies or psychological.

This case report provides the individualized effective treatment using radiation therapy in a patient with recurrent hidradenitis suppurativa where other treatments fail.

\section{Case Report}

A 26-year-old female without any chronic disease, presented recurrent painful, pus filled carbuncles at both axillae for 5 years. Because the symptoms were sporadic and firstly subsided by themselves, she did not seek any medical care. She experienced this condition for about one year. The disease condition worsened so she decided to seek medical treatment. The diagnosis was Hurley

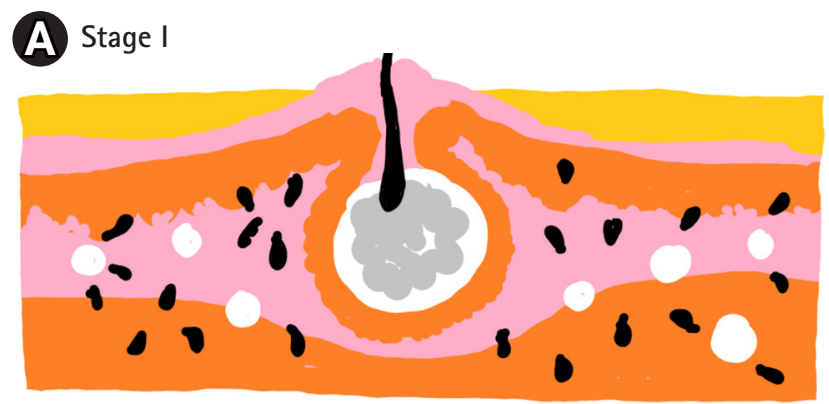

B Stage II

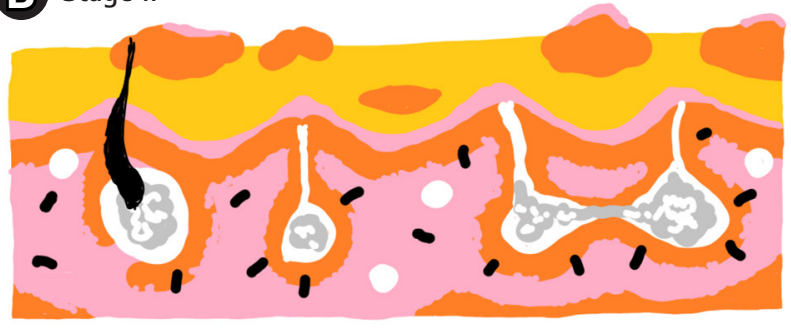

(C) Stage III

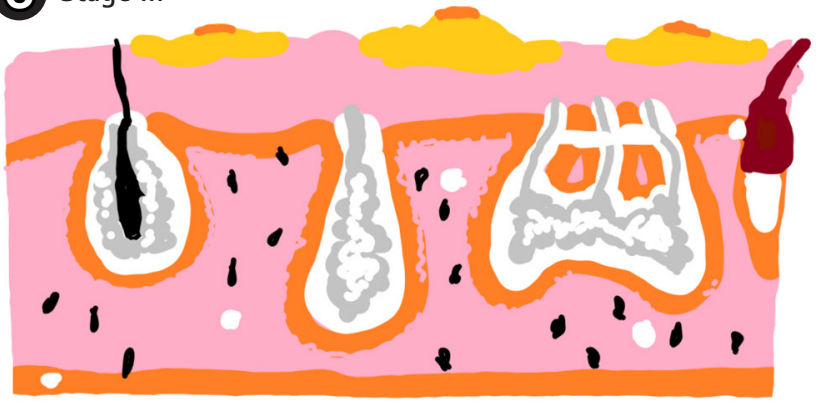

Fig. 1. Hurley stage. (A) Stage I consists of solitary or multiple isolated abscesses without scarring or sinus tract formation. (B) Stage II is characterized by single or multiple recurrent abscesses with widely separated lesions and sinus tract formation. (C) Stage III is characterized by diffuse involvement across a region with multiple interconnected sinus tracts and abscesses. stage III hidradenitis suppurativa at the left axilla (Fig. 2) and stage II at the right axilla (Fig. 3). All author declare that written informed consent was obtained from the patient for publication of this case report and accompanying image.

\section{Previous treatments}

In both axillae, she was treated using topical antibiotics (clindamycin lotion 1\%) and oral antibiotics (rifampicin and clindamycin). Her symptoms worsened and spread beyond those regions. Pain and infections also worsened causing offensive odors from the lesions.

Injecting triamcinolone acetonide in the hidradenitis suppurativa lesions has been used combined with oral antibiotics and oral isotretinoin. After treatment, her inflammation and pain slightly subsided. Unfortunately, the purulent nodules recurred in 2 months. Excision with drainage was designed as the next treatment of her both axillae, and intravenous antibiotics were used during the admission for surgery.

At postoperative follow-up, the right axilla showed only scar formation without any infection. The left axilla revealed some improvement but with frequent recurrence. Multiple wide excisions were performed thereafter at the left axilla indicating similar pathologic findings of chronic inflammation. Isotretinoin was still provided but proved to be less effective.

At this moment, her quality of life worsened. Some carbuncles slightly increased with infected ulcers at the left axilla. She socially isolated herself because of embarrassment from her disease. Because the disease continued to worsen, she was referred for radiation therapy (Fig. 2).

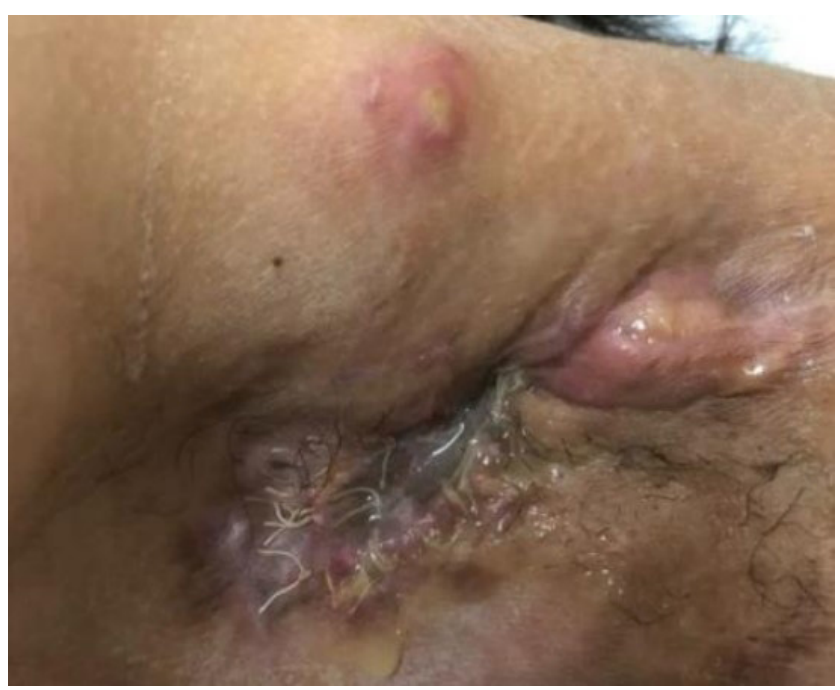

Fig. 2. Hurley stage III at left axilla (pre radiation therapy). There were multiple interconnected sinus tracts and abscesses with foul odor at left axilla. She suffered from the pain at this area. 


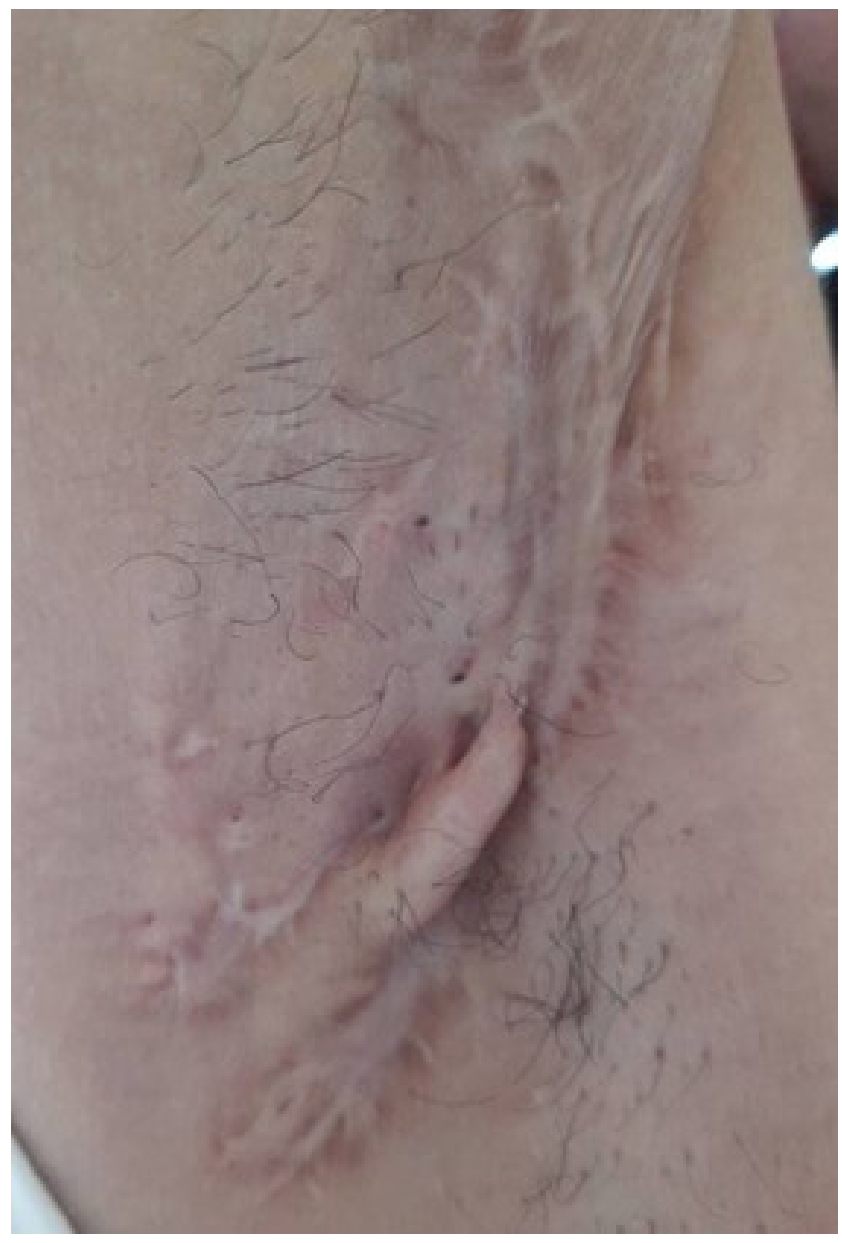

Fig. 3. Hurley stage II at right axilla. There are previously multiple recurrent abscesses with widely separated lesions and sinus tract formation at right axilla. She had both medical and surgical treatment at right axilla. The scar formation was seen after treatment. There were no any abscess or pus tract.

\section{Radiation therapy}

Computed tomography (CT) simulation was performed in the supine position. Adduction of the left arm was performed. The CT scan found ulcers, pus cavities and deep chronic pus tracts at her left axilla (Fig. 4), and metal wire was used to localize the boundary of disease.

CT images were imported to Eclipse to define the targets and organ-at-risk. Ulcer, pus cavities, pus tracts and axillar skin were all contoured in the gross target volume (GTV) to guide the radiation therapy field. A 1-cm margin was added in all directions from the GTV to the planning target volume (PTV). Eclipse treatment planning system (TPS) version 15.6 (Varian Medical Systems, Palo Alto, CA, USA) was used.

Radiation therapy was provided by three-dimensional conformal radiation therapy using $6 \mathrm{MV}$ photons with opposing anterior-posterior fields, $7.5 \mathrm{~Gy}$ in 3 fractions (2.5 Gy/fraction). Maximum avail-
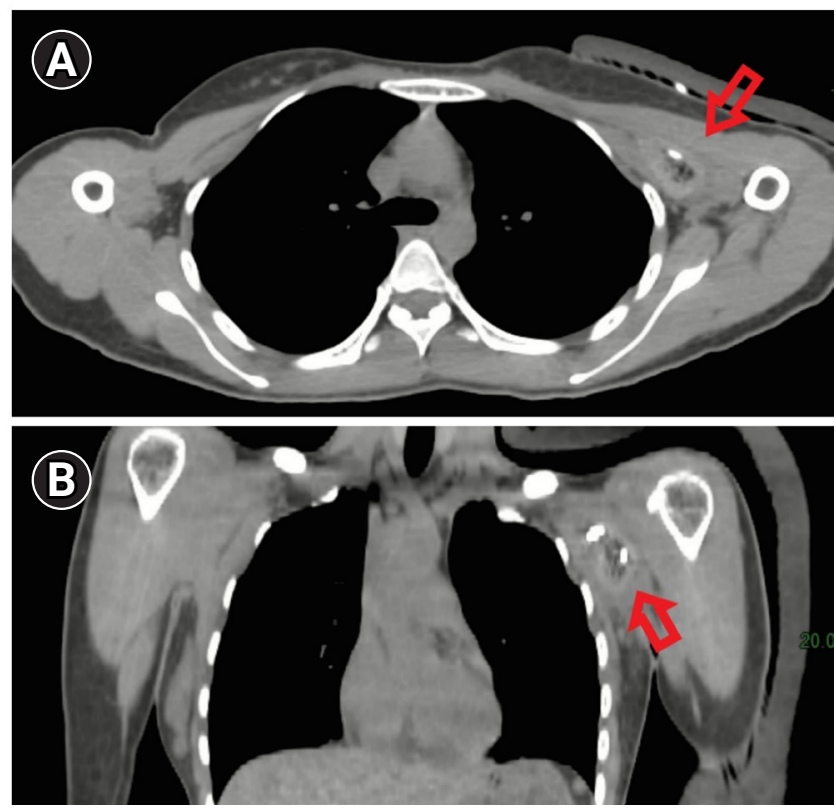

Fig. 4. Computed tomography (CT) simulation: $(A)$ axial view and $(B)$ coronal view. The CT simulation scan found ulcers, pus cavities and deep chronic pus tracts at her left axilla (arrow).

able dose rate was $400 \mathrm{MU} / \mathrm{min}$. Dose distribution was calculated by the anisotropic analytical algorithm. Each field was shaped by conforming the multi-leaf collimator to the PTV. A 1-cm thickness skin bolus was placed covering all radiation therapy areas during every fraction. Treatment plans included a single isocenter placed at the center of the PTV. The 3 fractions of radiation therapy were provided on November 7, 9, and 10, 2020 (Fig. 5).

In treatment room, patient was set similar to the simulation position. The MV portal for standard planar imaging with Elekta Synergy Platform (Elekta $A B$, Stockholm, Sweden) was used as image guided radiation therapy in every fraction before radiation therapy treatment.

\section{Treatment results}

Evaluating on the last day (3rd fraction) of radiation therapy, the carbuncles and wounds at the axilla had much improved. The pus subsided, pain was relieved, and less odor from the lesions was perceived. No erythematous skin or wound after radiation therapy was noted (Fig. 6).

At 3-month follow-up in March 2021, the skin of the axilla was smoother and dry. No pus or carbuncles were detected. Some granulation tissue was seen at her left axilla without foul odor (Fig. 6).

\section{Discussion}

Hidradenitis suppurativa most commonly presents after puberty. In 


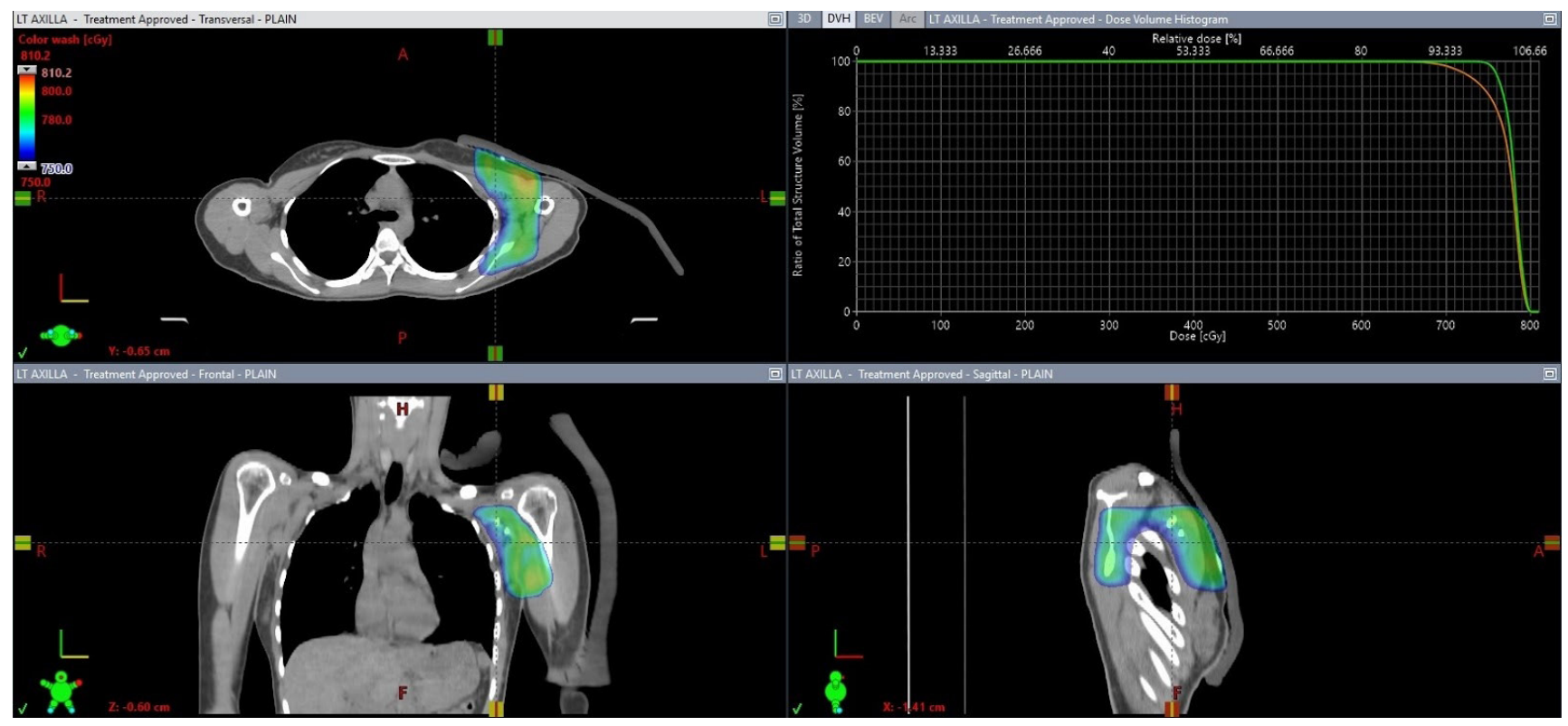

Fig. 5. Total radiation dose in three-dimensional conformal radiation therapy planning of 7.5 Gy in 3 fractions at left axilla lesion. Green line represented gross target volume coverage (maximum dose, $8.1 \mathrm{~Gy}$; mean dose, $7.8 \mathrm{~Gy}$ ). Orange line represented planning target volume coverage (maximum dose, $8.1 \mathrm{~Gy}$; mean dose, $7.7 \mathrm{~Gy}$ ).

most patients, flares are accompanied by increasing pain symptoms and suppuration at varying periods, often occurring among premenstrual women. When untreated, flares may subside within 7 to 10 days.

The prevalence of this disease from a French community-based study [6] showed 1\% occurrence over 1 year. Some studies, including young adults (18 to 33 years of age) related to sexually transmitted diseases, have shown a point prevalence of up to $4 \%$ [7]. Women are more frequently affected than men (3:1), and this disease commonly develops in the early 20s. Nevertheless, the onset of this condition may develop in prepubertal children and postmenopausal women as well [6].

Autosomal dominant inheritance relates to one-third of patients with hidradenitis suppurativa. Some patients present severe acne and perifolliculitis capitis which has been linked to chromosome 1p21.1-1q25.3 and mutations of the $\gamma$-secretase complex [8].

Arthritis (rheumatoid factor negative and HLA-B27 negative), usually occurring at the peripheral joints, appears more frequent among patients with hidradenitis suppurativa than that in general population [9].

Other dermatologic and non-dermatologic conditions such as inflammatory bowel disease, SAPHO syndrome (synovitis, acne, palmoplantar pustulosis, hyperostosis, osteitis), pyoderma gangrenosum, Adamantiades-Behçet disease, spondyloarthropathy, keratitis-ichthyosis-deafness syndrome, Down's syndrome, squamous cell carcinoma, adenocarcinoma, acne conglobata, severe acne and pi-
Ionidal cysts are all associated with hidradenitis suppurativa [9].

Smoking and obesity are risk factors for developing hidradenitis suppurativa and disease severity [10].

This disease has a substantial negative impact on the quality of life of the patients compared with that in the general population. Higher sick leaves from work and low scores on the self-reported level of health status scales were reported among these patients [11].

\section{Etiology, pathogenesis}

The etiology of hidradenitis suppurativa remains unclear. Hidradenitis suppurativa is a disease of the hair follicles. One study suggested that it constitutes a multifocal disease, in which atrophy of the sebaceous glands occurs followed by early lymphocytic inflammation and hyperkeratosis of the pilosebaceous unit. Hair follicle destruction and granuloma formation subsequently occurred [12]. Scarring and sinus tract formation stem from the healing process.

Some studies have suggested that the interleukin-12 to interleukin-23 pathway and tumor necrosis factor a (TNF-a) are involved in the pathogenesis of hidradenitis suppurativa, supporting the proposition that it comprises an immune or inflammatory disorder $[13,14]$

\section{Treatments}

Treatment in hidradenitis suppurativa is categorized as preventive, pharmacologic, surgical, or psychological. Preventive treatment in- 


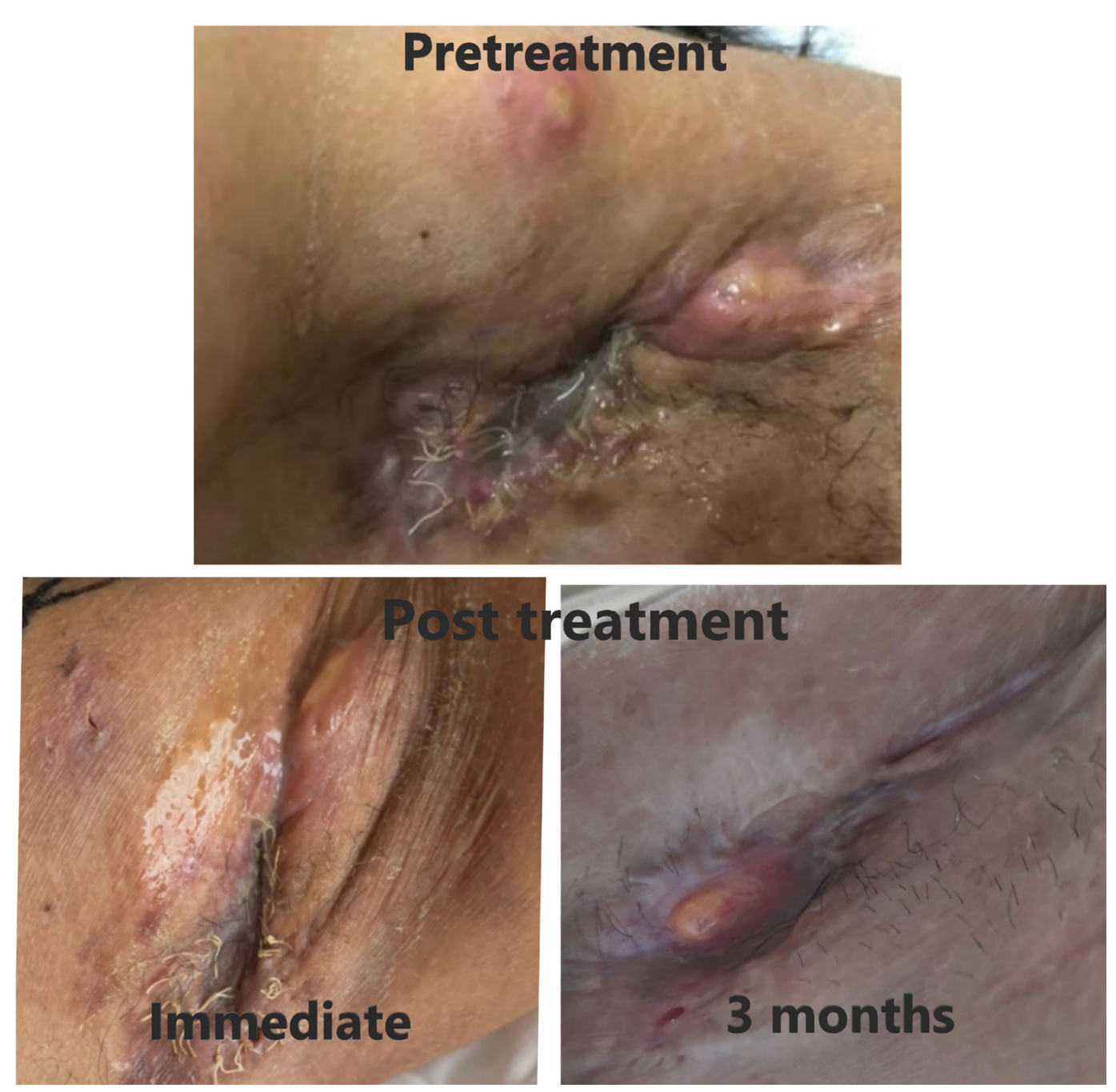

Fig. 6. Post-treatment outcome of left axilla compared between pretreatment, immediate post-treatment (3rd fraction), and 3 months post-treatment. Immediate post-treatment (at 3rd fraction), the carbuncles and wound at the axilla had much improvement. The pus subsided. The pain was relieved. Less odor from the lesions was noticed. There is no erythematous skin or wound after radiation therapy. The 3 months post-radiation therapy, the skin of the axilla was smoother and dry. No pus or carbuncles were detected. Some granulation tissue was seen at her left axilla. There is no foul odor.

volves losing weight, ceasing smoking, avoiding shaving, avoiding tight clothing, managing stress, and using warm compresses. No clear guidelines are available on conservative management, but it may be used in the early stage I hidradenitis suppurativa [10]. Pharmacologic therapy includes topical antibiotics (i.e., topical clindamycin, etc.), systemic antibiotics (i.e., rifampicin, clindamycin, etc.), intralesional corticosteroids, hormonal therapy, retinoids, immunosuppressants (i.e., cyclosporine, TNF inhibitors, etc.), and biologic agents [15]. Surgical intervention is preferred as a last option for unresponsive lesions. Patients with stage I and II hidradenitis suppurativa do not need surgical interventions. Wide excision of all hair-bearing areas in the affected region provides much higher benefits compared with excision of just the inflamed lesions [16].

Local therapies include cryotherapy, neodymium: yttrium-alumi- num-garnet laser therapy, and radiation therapy may be applied. Newer therapies such as carbon dioxide laser and the use of anti-inflammatory drugs such as infliximab combined with surgery have shown the good outcomes [17]. However, in this study, some patients needed to be treated under general or local anesthesia which presented some difficulty in performing. Apart from that, axillary scar contracture complication was observed in two patients limiting the patients' movement. Moreover, only some centers could provide these treatments. Therefore, from these treatment options of hidradenitis suppurativa, some limited knowledge of effectiveness and complications remain.

This study comprises a case report regarding radiation therapy in hidradenitis suppurativa, Hurley stage III in the left axillary site. Other case report studies of radiation therapy in hidradenitis sup- 
purativa all used electron beam therapy with varied radiation doses. Trombetta et al. [18] reported on patients with axillary and inguinal hidradenitis suppurativa without details of disease staging. The results showed good response to electron beam radiation therapy with no evidence of recurrence at 2.75 years or evidence of toxicity [18]. In other series such as from Frohlich et al. [19] of 231 German patients treated with radiation therapy without site-specific data, the complete response rate was 89 (38.5\%), whereas 92 (39.8\%) showed some improvement in symptoms. Therefore, it remains unclear whether any different response rate was found between the different sites. However, in the site using only axillary radiation therapy, the study suggested the effectiveness of using radiation therapy [18].

Various dose fractionations were reported in many studies. The optimal dose fractionation scheme remains unclear and may differ at different sites. According to Trombetta et al. [18], the most common dose reported with good local control of disease in many studies was $7.5 \mathrm{~Gy}$ in 3 fractions using electron therapy. It remains unclear whether escalating or reducing dose would result in optimal response rates. Evaluation in a prospective design is needed to determine optimal fraction schedules.

Concerning the reduced inflammation effects after radiation therapy, some studies have supported using radiation therapy to inhibit the proinflammatory pathway (NF-kB, STAT). It could possibly decrease inflammation in this case [20].

Our case report study showed a good outcome using photon beam radiation therapy in hidradenitis suppurativa. An early response was observed in the third fraction of radiation therapy helping to relieve many symptoms. Long-term disease control was the other benefit. We used CT simulation to evaluate the extension of disease. We observed that apart from the physical exam, some obscure diseases were deeply seated inside. For example, some long sinus tracts might easily not have been covered in the radiation field. Thus, CT simulation proved a helpful tool in defining the entire disease burden. We used 6 MV photon therapy instead of electron therapy because of the awareness of the need to cover the deep-seated lesions. In the photon planning we contoured all of the disease extensions and observed all the plan coverage. No acute toxicity occurred during radiation therapy or post-treatment with short follow-up. Nevertheless, one important issue to discuss with the patient was the stochastic effects of radiation, including carcinogenic effects when using low doses of radiation therapy in benign disease.

However, radiation therapy may play a role in chronic hidradenitis suppurativa, especially among patients with axillary disease or cases of refractory hidradenitis suppurativa. A prospective study is necessary to further evaluate the disease response, optimal dose fractionations, and the risk of long-term toxicity.

In conclusion, apart from other common treatment modalities in hidradenitis suppurativa, Hurley stage III, radiation therapy using photon beam, 7.5 Gy in 3 fractions (2.5 Gy/fraction) proved effective treatment. Radiation therapy showed early treatment response and good disease control improving patient quality of life and served as one treatment option of this disease.

\section{Conflict of Interest}

No potential conflict of interest relevant to this article was reported.

\section{Acknowledgements}

Firstly, I would like to express my sincere gratitude to my Prof. Prasert Lertsanguansinchai for the continuous support of my study and related research, for his patience, motivation, and immense knowledge. His guidance helped.

In addition, I would like to express my sincere thanks to other colleagues, Dr. Thirada Vongvanichvathana and our nurses for their help and encouragement throughout the course of this research.

\section{References}

1. Mebazaa A, Ben Hadid R, Cheikh Rouhou R, et al. Hidradenitis suppurativa: a disease with male predominance in Tunisia. Acta Dermatovenerol Alp Pannonica Adriat 2009;18:165-72.

2. Lapins J, Jarstrand C, Emtestam L. Coagulase-negative staphylococci are the most common bacteria found in cultures from the deep portions of hidradenitis suppurativa lesions, as obtained by carbon dioxide laser surgery. Br J Dermatol 1999;140:90-5.

3. Wortsman $X$, Jemec GB. Real-time compound imaging ultrasound of hidradenitis suppurativa. Dermatol Surg 2007;33:13402.

4. Hurley HJ. Axillary hyperhidrosis, apocrine bromhidrosis, hidradenitis suppurativa, and familial benign pemphigus: surgical approach. In: Roenigk RK, Roenigk HH, editors. Roenigk \& Roenigk's dermatologic surgery: principles and practice. 2nd ed. New York, NY: Marcel Dekker; 1996, p. 623-45.

5. Saunte DM, Jemec GB. Hidradenitis suppurativa: advances in diagnosis and treatment. JAMA 2017;318:2019-32.

6. Revuz JE, Canoui-Poitrine F, Wolkenstein P, et al. Prevalence and factors associated with hidradenitis suppurativa: results from two case-control studies. J Am Acad Dermatol 2008;59:596601.

7. Jemec GB, Heidenheim M, Nielsen NH. The prevalence of hidrad- 
enitis suppurativa and its potential precursor lesions. J Am Acad Dermatol 1996:35(2 Pt 1):191-4.

8. Wang $B$, Yang W, Wen $W$, et al. Gamma-secretase gene mutations in familial acne inversa. Science 2010;330:1065.

9. Jemec GB. Clinical practice: hidradenitis suppurativa. N Engl J Med 2012;366:158-64.

10. Sartorius K, Emtestam L, Jemec GB, Lapins J. Objective scoring of hidradenitis suppurativa reflecting the role of tobacco smoking and obesity. Br J Dermatol 2009;161:831-9.

11. Esmann S, Jemec GB. Psychosocial impact of hidradenitis suppurativa: a qualitative study. Acta Derm Venereol 2011;91:328-32.

12. Yu CC, Cook MG. Hidradenitis suppurativa: a disease of follicular epithelium, rather than apocrine glands. Br J Dermatol 1990;122: 763-9.

13. Matusiak L, Bieniek A, Szepietowski JC. Increased serum tumour necrosis factor-alpha in hidradenitis suppurativa patients: is there a basis for treatment with anti-tumour necrosis factor-alpha agents? Acta Derm Venereol 2009;89:601-3.

14. Schlapbach C, Hanni T, Yawalkar N, Hunger RE. Expression of the IL-23/Th17 pathway in lesions of hidradenitis suppurativa. J Am
Acad Dermatol 2011;65:790-8.

15. Shah N. Hidradenitis suppurativa: a treatment challenge. Am Fam Physician 2005;72:1547-52.

16.Mandal A, Watson J. Experience with different treatment modules in hidradenitis suppuritiva: a study of 106 cases. Surgeon 2005;3:23-6.

17. Madan V, Hindle $E_{1}$ Hussain W, August PJ. Outcomes of treatment of nine cases of recalcitrant severe hidradenitis suppurativa with carbon dioxide laser. Br J Dermatol 2008;159:1309-14.

18. Trombetta M, Werts ED, Parda D. The role of radiotherapy in the treatment of hidradenitis suppurativa: case report and review of the literature. Dermatol Online J 2010;16:16.

19. Frohlich D, Baaske D, Glatzel M. Radiotherapy of hidradenitis suppurativa: still valid today? Strahlenther Onkol 2000;176:2869.

20. Yu Jl, Park HC, Choi DH, et al. Prospective phase II trial of regional hyperthermia and whole liver irradiation for numerous chemorefractory liver metastases from colorectal cancer. Radiat Oncol J 2016;34:34-44. 